\title{
Dynamics of radiatively inefficient flows accreting onto radiatively efficient black hole objects
}

\author{
Daniel Proga \\ Department Physics, University of Nevada, Las Vegas, Las Vegas, NV 89154, USA \\ email: dproga@physics.unlv.edu
}

\begin{abstract}
I present results from numerical simulations of gas dynamics outside luminous accretion disks in active galactic nuclei. The gas, gravitationally captured by a super massive black hole, can be driven away by the energy and momentum of the radiation emitted during black hole accretion. Assuming axisymmetry, I study how the mass accretion and outflow rates, and the flow dynamics respond to changes in radiation heating relative to radiation pressure.

I find that for a $10^{8} \mathrm{M}_{\odot}$ black hole with the accretion luminosity of 0.6 of the Eddington luminosity the flow settles into a steady state and has two components: (1) an equatorial inflow and (2) a bipolar inflow/outflow with the outflow leaving the system along the disk rotational axis. The inflow is a realization of a Bondi-like accretion flow. The second component is an example of a non-radial accretion flow which becomes an outflow once it is pushed close to the rotational axis where thermal expansion and radiation pressure accelerate it outward.

The main result of this preliminary work is that although the above two-component solution is robust, its properties are sensitive to the geometry and spectral energy distribution of the radiation field.
\end{abstract}

Keywords. Accretion, accretion disks - methods: numerical - hydrodynamics

\section{Introduction}

The radiation properties of active galactic nuclei (AGN) and the AGN central location in their host galaxies imply that they play a very important role in determining the ionization structure and dynamics of matter not only in their vicinity but also on larger, galactic and even intergalactic scales (Cotti \& Ostriker, 1997, 2001; King 2003; Murray, Quataert, \& Thompson 2005; Sazonov et al. 2005; Springel, Di Matteo \& Hernquist 2005; Hopkins et al. 2005, and references therin). Many observational results support this suggestion, in particular the presence of broad emission and absorption lines in AGN spectra. The ionization structure and dynamics of the gas responsible for these lines can be driven by radiation, even for sub-Eddington sources. The driving can be due to radiation pressure or radiation heating, or both (e.g., Begelman, McKee and Shields, 1982; Shlosman, Vitello \& Shaviv 1985; Ostriker, McKee, \& Klein 1991; Arav \& Li 1994; Murray et al. 1995; Proga, Stone \& Kallman 2000; Proga \& Kallman 2002, 2004).

In this paper, I present results from hydrodynamical simulations of a non-rotating gas on sub-parsec- and parsec-scales in AGNs. I use a simplified version of the numerical method developed by Proga, Stone \& Kallman (2000) to study a related problem of radiation driven disk winds in AGN (for details see Proga et al. 2006 in preparation). I consider an axisymmetric flow accreting onto a supermassive black hole (BH). The flow is non-spherical because it is irradiated by an accretion disk. The disk radiation flux is the highest along the disk rotational axis and is gradually decreasing with increasing polar angle, $\theta$ as $\cos \theta$. The flow is also irradiated by an isotropic corona (see eq. 2.1). I 
take into account the radiation heating and cooling, radiation pressure due to the electron scattering and spectral lines. I adopt a simplified treatment of photoionization, and radiative cooling and heating allowing for a self-consistent calculation of the ionization state, and therefore the line force, in the flow.

\section{Results}

I assume the mass of the non-rotating $\mathrm{BH}, M_{\mathrm{BH}}=10^{8} \mathrm{M}_{\odot}$ and the disk inner radius, $r_{*}=3 r_{\mathrm{S}}=8.8 \times 10^{13} \mathrm{~cm}$ throughout this paper. I consider the case with the rest mass conversion efficient $\eta=0.0833$ and the mass accretion rate, $\dot{M}_{\mathrm{a}}=10^{26} \mathrm{~g} \mathrm{~s}^{-1}$ $\left(=1.6 \mathrm{M}_{\odot} \mathrm{yr}^{-1}\right)$. These system parameters yield the accretion luminosity, $L=7.5 \times$ $10^{45} \mathrm{erg} \mathrm{s}^{-1}$, corresponding to 0.6 of the Eddington luminosity. To determine the radiation field, I specify the fraction of $L$ in the UV and X-ray band, as $f_{\mathrm{UV}}$ and $f_{\mathrm{X}}$, respectively.

Although, a quasar is powered by accretion, the disk accretion rate, that determines radiation, is not coupled to the rate, $\dot{M}_{\mathrm{in}}\left(r_{\mathrm{i}}\right)$ at which the non-rotating gas leaves the computational domain of these simulations through the inner boundary. This $\dot{M}_{\mathrm{a}}-\dot{M}_{\mathrm{in}}\left(r_{\mathrm{i}}\right)$ decoupling is physically motivated because an accretion disk is build from rotating gas but non-rotating gas will not significantly contribute to the disk mass and to the system luminosity. In this sense, I study radiatively inefficient flows accreting onto an object with a radiatively efficient accretion disk.

I present here preliminary results from simulations where all model parameters are fixed except for $f_{\mathrm{UV}}$ and $f_{\mathrm{X}}$. I consider three cases: case A with $f_{\mathrm{UV}}=0.5$ and $f_{\mathrm{X}}=0.5$, case $\mathrm{B}$ with $f_{\mathrm{UV}}=0.8$ and $f_{\mathrm{X}}=0.2$, and case $\mathrm{C}$ with $f_{\mathrm{UV}}=0.95$ and $f_{\mathrm{X}}=0.05$ ( Table 1 for summary of the runs).

At large radii, the radial radiation force from the disk and spherical corona can be approximated as

$$
F_{r}^{\mathrm{rad}}(r, \theta)=\frac{\sigma_{e} L}{4 \pi r^{2} c}\left[2 \cos \theta f_{\mathrm{UV}}(1+M(t))+f_{\mathrm{X}}\right]
$$

where $r$ and $\theta$ are the radius and polar angle in the spherical polar coordinate system, respectively while the terms in the brackets with $f_{\mathrm{UV}}$ and $f_{\mathrm{X}}$ correspond to the disk and corona contribution, respectively. For simplicity, I assume that all UV photons are emitted by the disk whereas all X-rays are emitted by the corona. The $M(t)$ is the socalled force multiplier - the numerical factor which parameterizes by how much spectral lines increase the scattering coefficient compared to the electron scattering coefficient (Castor, Abbott \& Klein 1975).

Figure 1 compares the results from run A and run C. I specified the outer boundary in the following way. The density and temperature at the outer radius, $r_{\mathrm{o}}$ was set to $\rho_{0}=10^{-21} \mathrm{~g} \mathrm{~cm}^{-3}$ and $T_{0}=2 \times 10^{7} \mathrm{~K}$, respectively. The velocity was set to zero. At the outer radial boundary, during the evolution of each model I continue to apply these constraints that the density and temperature are fixed at constant values at all times. For the initial conditions, I set all variables constant and equal to their values at the outer boundary, as listed above. The figure shows the instantaneous density, temperature, and distributions and the poloidal velocity field of the models. Additionally, it also shows the so-called Compton radius corrected for the effects of radiation pressure due to electron scattering

$$
\bar{R}_{\mathrm{C}} \equiv R_{\mathrm{C}}\left[1-\Gamma\left(2 \cos \theta f_{\mathrm{UV}}+f_{\mathrm{X}}\right)\right]
$$

where $R_{\mathrm{C}} \equiv G M_{\mathrm{BH}} \mu m_{p} / k T_{\mathrm{C}}=8.03 \times 10^{18} \mathrm{~cm}=9.1 \times 10^{4} r_{*}$ is the uncorrected Compton radius for the Compton temperature, $T_{\mathrm{C}}=2 \times 10^{7} \mathrm{~K}$. 

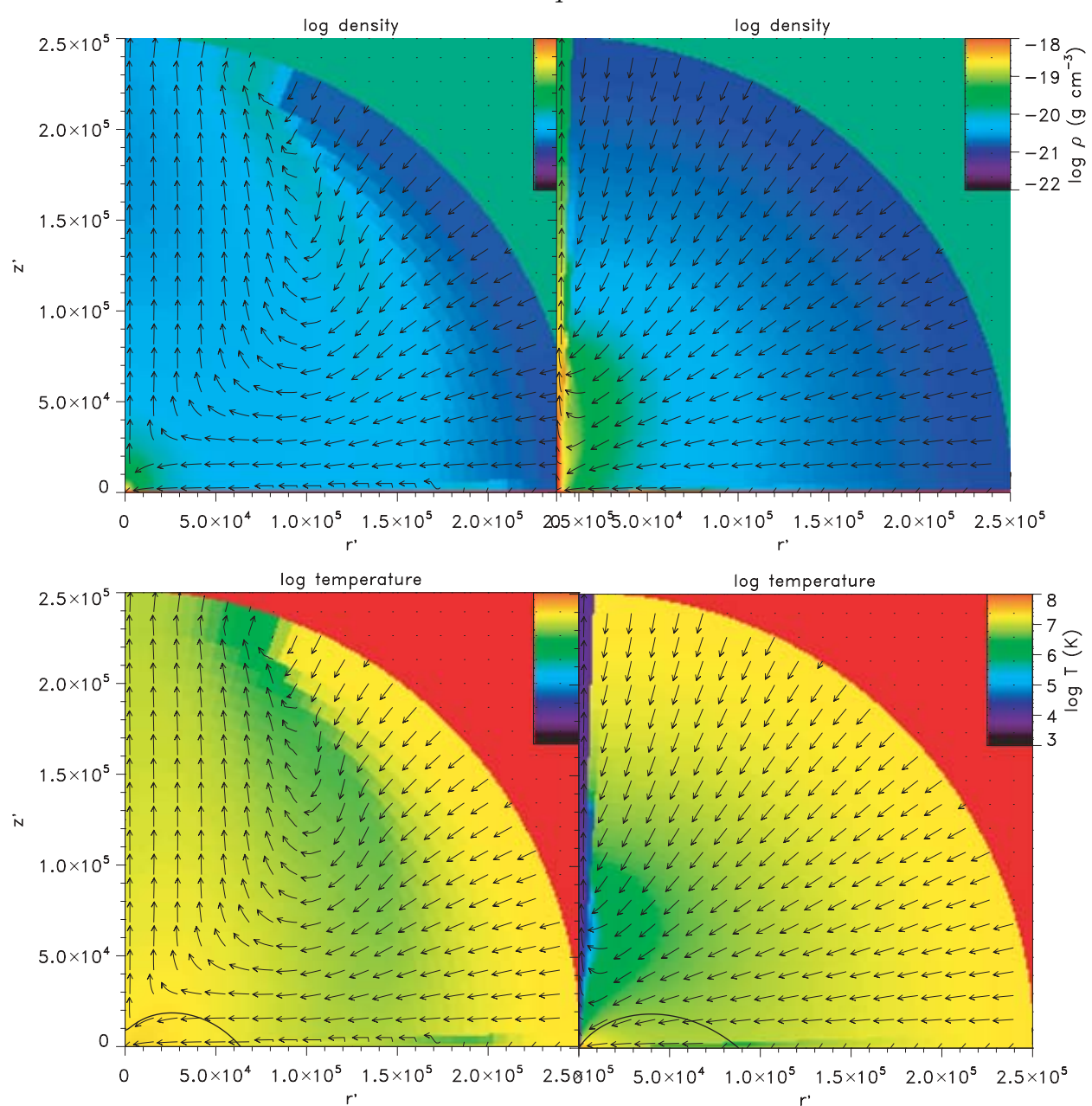

Figure 1. Comparison of the results for run A and C (left and and right column, respectively). Top row of panels: Maps of logarithmic density over-plotted by the direction of the poloidal velocity. Bottom row of panels: Maps of logarithmic temperature over-plotted by the direction of the poloidal velocity. The solid curve in the bottom left corner marks the position of the Compton radius corrected for the effects of radiation pressure due to electron scattering (see eq. 2.2 in the main text). The length scale is in units of the inner disk radius (i.e., $r^{\prime}=r / r_{*}$ and $z^{\prime}=z / r_{*}$. The computational domain is defined to occupy the angular range $0^{\circ} \leqslant \theta \leqslant 90^{\circ}$ and the radial range $r_{\mathrm{i}}=500 r_{*} \leqslant r \leqslant r_{\mathrm{o}}=2.5 \times 10^{5} r_{*}$.

In all runs, the flow settles quickly into a steady state (within $\sim$ a few $\times 10^{12} \mathrm{~s}$ which correspond to a few dynamical time scales at $\left.r_{\mathrm{o}}, \tau=\left(r_{o}^{3} / G M_{\mathrm{BH}}\right)^{1 / 2}=9 \times 10^{11} \mathrm{~s}\right)$. The steady state consists of two flow components (1) an equatorial inflow and (2) a bipolar inflow/outflow with the outflow leaving the system along the pole. The outflow is collimated by the inflow. The calculations capture the subsonic and supersonic parts of both the inflow and outflow. Although the same components can be identified in all runs, their size, density and temperature, and the degree of outflow collimation depend on $f_{\mathrm{UV}}$ and $f_{\mathrm{X}}$ (the spectral energy distribution and geometry of the radiation field). In particular, the outflow power (e.g., measured as the kinetic energy, $P_{\mathrm{k}}$, and thermal energy $P_{\text {th }}$ carried by the outflow) and the degree of collimation is higher for the model 
Table 1. Summary of results

\begin{tabular}{lcccccccc}
\hline Run & $f_{\mathrm{UV}}$ & $f_{\mathrm{X}}$ & $\dot{M}_{\mathrm{in}}\left(r_{\mathrm{o}}\right)$ & $\dot{M}_{\mathrm{in}}\left(r_{\mathrm{i}}\right)$ & $\dot{M}_{\mathrm{out}}\left(r_{\mathrm{o}}\right)$ & $v_{r}$ & $P_{\mathrm{k}}\left(\mathrm{r}_{o}\right)$ & $P_{\mathrm{t}}\left(\mathrm{r}_{o}\right)$ \\
\hline $\mathrm{A}$ & 0.5 & 0.5 & 4 & 1 & 3 & 700 & 2 & 4 \\
$\mathrm{~B}$ & 0.8 & 0.2 & 8 & 3 & 5 & 4000 & 100 & 1 \\
$\mathrm{C}$ & 0.95 & 0.05 & 9 & 1 & 8 & 6700 & 300 & 0.03 \\
\hline
\end{tabular}

The quantities in the table are in the following units: $\dot{M}_{\text {in }}\left(r_{\mathrm{o}}\right), \dot{M}_{\text {in }}\left(r_{\mathrm{i}}\right)$, and $\dot{M}_{\text {out }}\left(r_{\mathrm{o}}\right)$ are in units of $10^{25} \mathrm{~g} \mathrm{~s}^{-1}, v_{r}$ is in units of $\mathrm{km} \mathrm{s}^{-1}$, and $P_{\mathrm{k}}\left(r_{\mathrm{o}}\right)$ and $P_{\mathrm{t}}\left(r_{\mathrm{o}}\right)$ are in units of $10^{40} \mathrm{erg} \mathrm{s}^{-1}$.

with the radiation dominated by the UV/disk emission (run C) than for the model with the radiation dominated by the X-ray/corona emission (run A). I note that a very narrow outflow driven by radiation pressure on lines can carry more energy and mass than a broad outflow driven by thermal expansion (compared results for runs $\mathrm{C}$ and $\mathrm{A}$ ).

\section{Conclusions}

The simulations show that AGN can have a substantial outflow which originates from the inflow at large radii. Such an outflow can control the rate at which non-rotating matter is supplied to the AGN central engine because the outflow mass loss rate, $\dot{M}_{\text {out }}\left(r_{\mathrm{i}}\right)$, can be significantly higher than the mass inflow rate at small radii, $\dot{M}_{\text {in }}\left(r_{\mathrm{i}}\right)$. For example, in run $\mathrm{C}$, as little as $10 \%$ of the inflow at large radii reaches small radii because $90 \%$ of the inflow is turned into an outflow. However, even the power of the strongest outflow is very low compared to the radiation power (i.e., for run C, $P_{\mathrm{k}} / L=4 \times 10^{-4}$ ). Finally, the inflows and outflows, found in these simulations, can be related to material responsible for broad absorption and emission lines, and narrow absorption and emission lines observed in X-ray and UV spectra of AGN.

\section{Acknowledgements}

This work is supported by NASA through grants and HST-AR-10305 and HST-AR10680 from the Space Telescope Science Institute, which is operated by the Association of Universities for Research in Astronomy, Inc., under NASA contract NAS5-26555.

\section{References}

Arav, N. \& Li, Z. Y. 1994, ApJ, 427, 700

Begelman, M. C., McKee, C. F. \& Shields, G. A. 1983, 271, 30

Castor, J. I., Abbott, D. C., \& Klein, R. I. 1975, ApJ, 195, 157

Ciotti, L. \& Ostriker, J. P. 1997, ApJ, 487, L105

Ciotti, L. \& Ostriker, J. P. 2001, ApJ, 551, 131

Hopkins, P. F., Hernquist, L., Cox, T. J., Di Matteo, T., Martini, P., Robertson, B. \& Springel, V. 2005, ApJ, 630, 705

King, A. 2003, ApJ, 596, L27

Murray, N., Quataert, E. \& Thompson, T. A. 2005, ApJ, 618, 569

Ostriker, E. C., McKee, C. F. \& Klein, R. I. 1991, ApJ, 377, 5930

Proga, D. \& Kallman, T. R. 2002, ApJ, 565, 455

Proga, D. \& Kallman, T. R. 2004, ApJ, 616, 688

Proga, D., Stone, J. M. \& Kallman, T. R. 2000, ApJ, 543, 686

Sazonov, S. Y., Ostriker, J. P., Ciotti, L. \& Sunyaev, R. A. 2005, MNRAS, 358, 168

Shlosman, I., Vitello, P. A. \& Shaviv, G. 1985, ApJ, 294, 96

Springel, V., Di Matteo, T. \& Hernquist, L. 2005, ApJ, 620, L79 
Mitchell Begelman: Have you looked at what happens when you adjust heating versus radiation pressure? One would like to see to what extent heating inhibits accretion. That is, you want to see how much pre-heating is inhibiting the accretion flow, but you also have radiation pressure there... How close are you to the Eddington limit with this system?

DAniel Proga: The calculation was for 0.6 Eddington limit. The last example was actually a manifestation of unclear interplay between the two effects. I am currently in the middle of the parameter survey.

MAREK ABramowicz: What the gas does after it reaches the boundary of your computational domain?

DANIEL Proga: We do not consider that the gas will be interacting with the medium on larger scales, so in this model the gas will leave the system. I look at velocities at the outer radius, and they can be quite high - order of $10^{4} \mathrm{~km} / \mathrm{s}$. This exceeds the escape velocity from the system. 


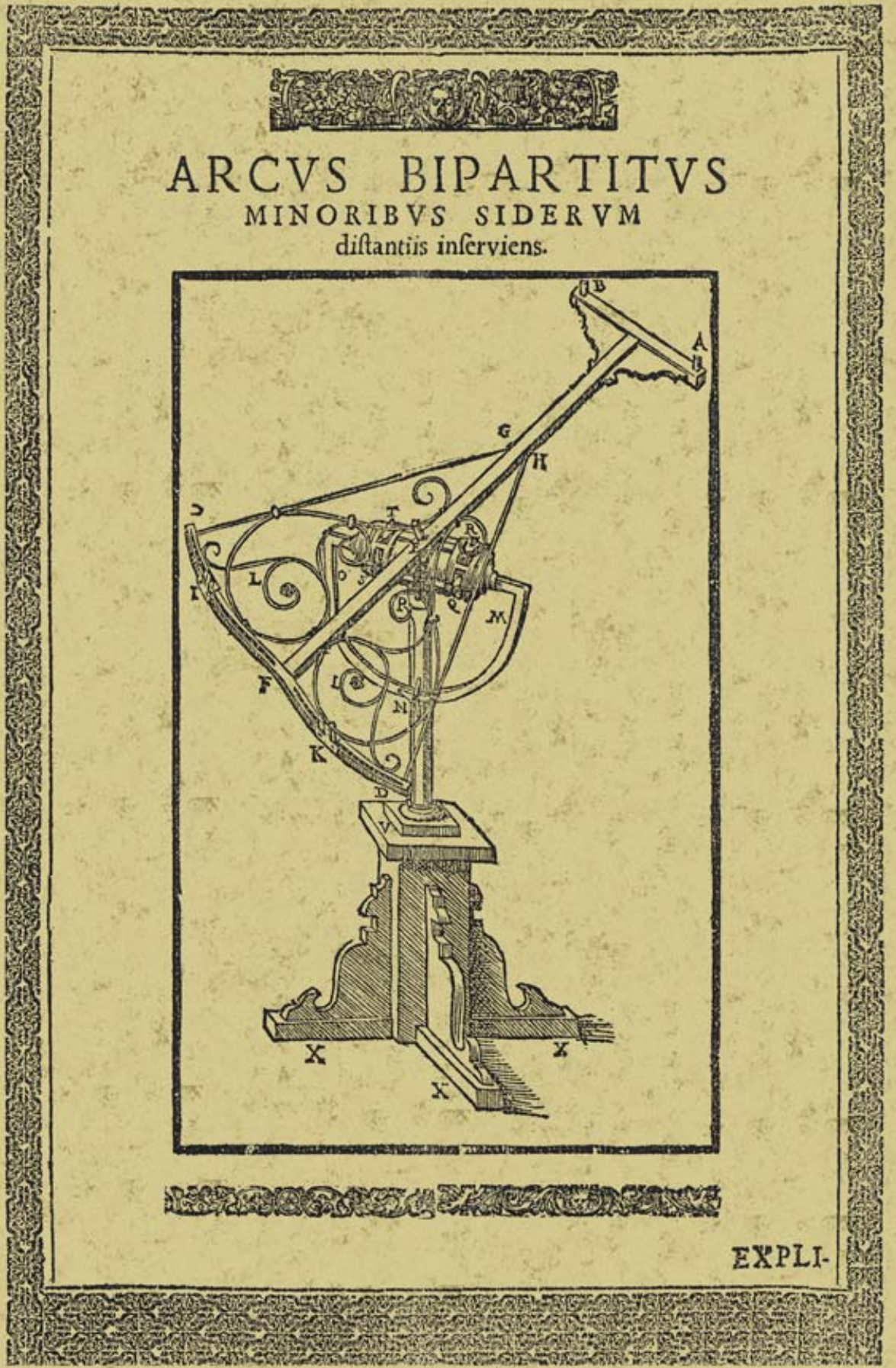

